\title{
Hubble's Constant and Flat Rotation Curves of Stars: Are Dark Matter and Energy Needed?
}

\author{
Alexandre Chaloum Elbeze \\ University Pierre et Marie Curie, Paris, France \\ Email: A77190@bezeqint.net
}

How to cite this paper: Elbeze, A.C. (2017) Hubble's Constant and Flat Rotation Curves of Stars: Are Dark Matter and Energy Needed? Journal of Modern Physics, 8, 24-34.

http://dx.doi.org/10.4236/jmp.2017.81003

Received: October 9, 2016

Accepted: January 1, 2017

Published: January 4, 2017

Copyright $\odot 2017$ by author and Scientific Research Publishing Inc. This work is licensed under the Creative Commons Attribution International License (CC BY 4.0).

http://creativecommons.org/licenses/by/4.0/

(c) (i) Open Access

\begin{abstract}
Although dark energy and dark matter have not yet been detected, they are believed to comprise the majority of the universe. Observations of the flat rotation curve of galaxies may be explained by dark matter and dark energy. This article, using Newton's laws and Einstein's theory of gravitation, shows that it is possible to define a new term, called $E_{0}$, variable in time and space, of which one of its limits is the Hubble constant $H_{0}$. I show that $E_{0}$ is strongly linked to an explanation of the flat rotation curve of galaxies. This strong correlation between Hubble's constant $H_{0}$ and $E_{0}$ enables us to solve the mystery of the surplus of gravity that is stabilizing the universe.
\end{abstract}

\section{Keywords}

Hubble's Law, General Relativity, Cosmological Constant, Expanding Universe, Dark Energy

\section{Introduction}

Galaxies appear to rotate more quickly than allowed by the gravity of its observable matter. Logically, they should have been destroyed a long time ago, as should galaxy clusters. Because of this disagreement between theory and observations, some undetectable element is believed to be providing galaxies with the additional mass that they need to avoid breaking up. This mysterious element has been called "dark matter". Over the years, coherent theories on dark matter and dark energy have been formulated to explain the stability of galactic systems. Attempts have been made to modify Newton's laws of gravity, for example, Milgrom's MOND theory [1], which explains rather well the flat rotation curves of stars for many galaxies. However, these theories have not yet offered a sufficiently well explained source; they are rather empirical theories. In this article, using Newton's laws and Einstein's theory of general relativity [2], I show that a coherent theory is possible that can explain, at the same time, flat rotation curves, 
the expansion of the universe, and the acceleration of the expansion. I will define a new term, $E_{0}$, one limit of which is the Hubble constant, and I clearly show its relationship with the flat rotation curves of galaxies. This new term $E_{0}$ is universal because it applies without restriction to all celestial bodies.

\section{Hubble's Constant and Defining the New Term $E_{0} \approx H_{0}$}

The Hubble constant $H_{0}$ is the constant of proportionality between the distance and speed of apparent recession of galaxies relative to any point in the observable universe. It is connected to the famous Hubble's law, which describes the expansion of the universe. Although referred to as a constant, $H_{0}$ actually varies depending on time and distance. It thus describes the growth rate of the universe at one moment and at a given distance from the point of observation. The most precise value of $H_{0}$ (from optical observations, May 2001) is $72 \pm 8 \mathrm{~km} \cdot \mathrm{s}^{-1} \cdot \mathrm{Mpc}^{-1}$.

However, the current definition of $H_{0}$ does not relate expansion of the universe to the flat rotation curves of galaxies and galaxy clusters. I must thus extend the concept of expansion of the universe to a comparison of the gravitational acceleration for small and large distances. Thus Newtonian acceleration can be written as $\gamma_{\text {Newton }}$ for small distances (less than $\mathrm{kpc}$ ), and $\gamma_{\text {measured }}$ and $D_{\text {measured }}$ (for distances greater than kpc) are the measured acceleration and distance, respectively, between gravitationally interacting masses. We can therefore write the following relationship for a galactic system in gravitational balance:

$$
\gamma_{\text {measured }}=\frac{V_{\text {measured }}^{2}}{D}
$$

where $V_{\text {measured }}$ is the measured speed of rotation. Einstein's relativity theory may also be applied to Newtonian gravitational acceleration $\gamma_{\text {Newton }}$ so that the masses vary as $\frac{M}{\sqrt{1-\frac{v^{2}}{c^{2}}}}$ and the distances in the moving reference frame (for the observer) shorten according to $d \cdot \sqrt{1-\frac{v^{2}}{c^{2}}}$. In fact, Newton's second law in relativistic mechanics (special relativity) may be applicable in my calculations. Here, $v$ represents the radial velocity between two gravitationally interacting objects (not to be confused with the rotation speed $V_{\text {measured }}$ of planets, stars, galaxies, and galaxy clusters around the center of their respective system). This radial velocity $v$ can also be regarded as the recessional velocity of the object of interest (galaxies, stars, planets) similar to the relationship $H_{0} \cdot D$, where $H_{0}$ is the Hubble constant and $D$ is the distance.

On the other hand, I take into account the relativistic function of acceleration $\gamma$ developed by Elbeze [3] [4], in which I include the recessional rate of the universe defined by function $E_{0} \cdot D$ (added at the possible radial speed $v$ ), which will be defined below (and is equivalent to the Hubble expression $H_{0} \cdot D$ ), and obtain:

$$
\gamma_{\text {Newton }}=\frac{G \cdot M}{D^{2}} \cdot \frac{1}{\sqrt{1-(v+E o \cdot D) / c}} \cdot \text { and } V_{\text {Newton }}=\sqrt{\frac{G \cdot M}{D} \cdot \frac{1}{\sqrt{1-(v+E o \cdot D) / c}}}
$$


From these relationships, I can define a rate of variation of acceleration $\tau_{\gamma}$ between the Newtonian acceleration (for all distances) and the measured acceleration $\gamma_{\text {measured }}$ as follows:

$$
\tau_{\gamma}=\frac{\gamma_{\text {measured }}-\gamma_{\text {Newton }}}{\gamma_{\text {Newton }}}
$$

I can compare Equation (3) with the Hubble constant $H_{0}$ as follows:

$$
\tau_{\gamma} \cdot V_{0} \cdot \frac{1}{D}=E_{0} \approx H_{0}
$$

where $E_{0}$ is the equivalent of $H_{0}$ in this article, and $V_{0}$ is the reference radial velocity, which I will define below. By replacing accelerations $\gamma_{\text {measured }}$ and $\gamma_{\text {Newton }}$ with their equivalent in speed and distance in Equations (1), (2), (3), and (4), so that radial velocity is negligible in calculating the rate $E_{0} \cdot D$, while applying the relativistic principle for distance and masses in radial motion (defined above and according to Newton's second law in special relativity to a radial dimension along $\boldsymbol{D}$ ), and with $\beta=\left(1-\left(E_{0} \cdot D / c\right)^{2}\right)^{-\frac{1}{2}}$, I obtain:

$$
\frac{\frac{V_{\text {measured }}^{2}}{D}-\frac{\beta^{2} \cdot G \cdot \text { Mgalaxy }}{D^{2}}}{\frac{\beta^{2} \cdot G \cdot \text { Mgalaxy }}{D^{2}}} \cdot V_{0} \cdot \frac{1}{D}=E_{0}
$$

where $E_{0} \cdot D$ is the recessional speed of mass $M_{2}$ from mass $M_{1}$ (Figure 1 ).

Here, $D$ is the distance between masses $M_{1}$ and $M_{2}$, their relative speed $v$ (radial velocity) is negligible, and $V_{\text {measured }}$ is the measured speed of rotation of $M_{2}$ around mass $M_{1}$. Solving Equation (4) and Equation (5) for $E_{0}$ gives two universal solutions:

$$
E_{0}=\frac{1}{D} \cdot\left[ \pm c \cdot \sqrt{1-\left(\frac{V_{\text {Newton }}}{V_{\text {measured }}}\right)^{2}+\left(\frac{V_{\text {Newton }}}{V_{\text {measured }}}\right)^{4}\left(\frac{c}{2 V_{0}}\right)^{2}}-\left(\frac{V_{\text {Newton }}}{V_{\text {measured }}}\right)^{2} \frac{c^{2}}{2 V_{0}}\right]
$$

We can already assume that $M_{1}$ and the gravitational constant G (from equation 2) disappear from Equation (6), because they are factors in the elements $\left(\frac{V_{\text {Newton }}}{V_{\text {measured }}}\right)$ composing it. Only speeds $V_{\text {Newton }}$ and $V_{\text {measured }}$, distance $D$, and the reference speed $V_{0}$, which will be defined low, are present in Equation (6). Thus Equation (6) is universal, independent of $M_{1}$ and $M_{2}$, and applies to all points in the universe with no distinctions. Let us write the recessional velocities $v E_{0}$ and $v H_{0}$ as follows:

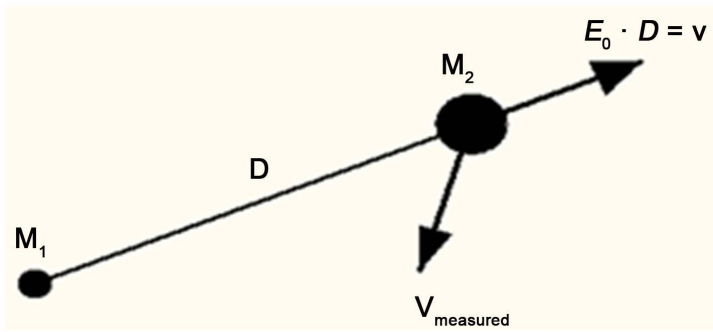

Figure 1. Measurement of the proposed recessional rate $E_{0}$ for coupled masses $M_{1}$ and $M_{2}$. 


$$
v E_{0}=E_{0} \cdot D \quad v H_{0}=H_{0} \cdot D
$$

\section{Relationship between $E_{0}$ and $H_{0}$ and the Flat Rotation Curves of Galaxies}

One possible solution for the speed of rotation $V_{\text {measured }}$, which solves Equation (6), is:

$$
V_{\text {measured }}=V E_{0}=\sqrt{\frac{G \cdot M_{g}}{D} \cdot\left[\frac{1}{\sqrt{1-\left(v+E_{0} \cdot D\right) / c}}\right] \cdot \Lambda\left[W p e \cdot \frac{D}{r h}+1\right]-\gamma D \cdot D}
$$

where $\gamma_{D}$ is the acceleration of the expansion of the universe (which is here regarded as small compared with gravitational acceleration $\gamma$ for distances $<1 \mathrm{Mpc}$ ); $G$ is Newton's gravitational constant; $M_{g}$ is the mass of the planet, star, galaxy, or galaxy cluster; $D$ and $v$ are the distance and relative radial speed, respectively, between masses $M_{1}$ and $M_{2}$ (Figure 1 ); $E_{0} \cdot D$ is the recessional velocity of the object of interest; $r h$ is the atomic radius of hydrogen; and the two functions $\Lambda$ (value between 1 and 2 ) and Wpe, which is on the order of $10^{-32}$ depending on the nature of the celestial body (e.g., galaxy, star), are defined in Appendix 1 and Appendix 2, respectively. By replacing $V_{\text {measured }}=V E_{0} \quad($ Equation $(8))$ in Equation (6), the term $\sqrt{\frac{G \cdot M}{D} \cdot \frac{1}{\sqrt{1-\left(v+E_{0} \cdot D\right) / c}}}$ from Equation (8) (which defines the Newtonian part of $V E_{0}$ ) disappears before the factors $\left(\frac{V_{\text {Newton }}}{V_{\text {measured }}}\right)$, so that with

$$
a(D)=\frac{1}{\sqrt{\Lambda \cdot\left(W p e \cdot \frac{D}{r h}+1\right)}}
$$

then Equation (5) becomes

$$
E_{0}=\frac{1}{D} \cdot\left[ \pm c \cdot \sqrt{1-a(D)^{2}+a(D)^{4}\left(\frac{C}{2 V_{0}}\right)^{2}}-a(D)^{2} \frac{c^{2}}{2 V_{0}}\right]
$$

I can apply Equation (8) (e.g., to our galaxy) in the case of gravitational balance of the Milky Way, and assume the acceleration of the expansion of the universe to be equal to $\gamma_{D}$ (defined low), here $\gamma_{D}$ is the acceleration in $\mathrm{m} \cdot \mathrm{s}^{-2}$, we obtain the Figures 2-4.

In Figure 2, the position of our Sun is at approximately $8.2 \mathrm{kpc}$ and its speed is $230 \mathrm{~km} \cdot \mathrm{s}^{-1}$. The speed returns to its Newtonian form to $2.5 \mathrm{Mpc}$. In my calculations, only the mass of the bulb is considered; I do not take account of the mass of the galactic gas clouds, which in any case do not influence the effective existence of the flat rotation curves of the galaxy, but modify only the form of the curve speeds. I apply Equation (9) to define $E_{0}$ (the proposed new expansion rate) in relation to the Hubble constant $H_{0}$ (Hubble expansion rate) (Figure 3).

Figure 3 shows four terms: $v E_{0}$ and $v H_{0}$, the proposed expansion rate $\left(E_{0}\right)$, and the Hubble constant $\left(H_{0}\right)$. Values of $E_{0}$ and $H_{0}$ are given in units of $\mathrm{km} \cdot \mathrm{s}^{-1} / \mathrm{Mpc}$; in this article, $H_{0}$ is equal to $73.0 \pm 1.75 \mathrm{~km} \cdot \mathrm{s}^{-1} / \mathrm{Mpc}$ for a distance of $D \approx 20 \mathrm{Mpc}$ [5] 


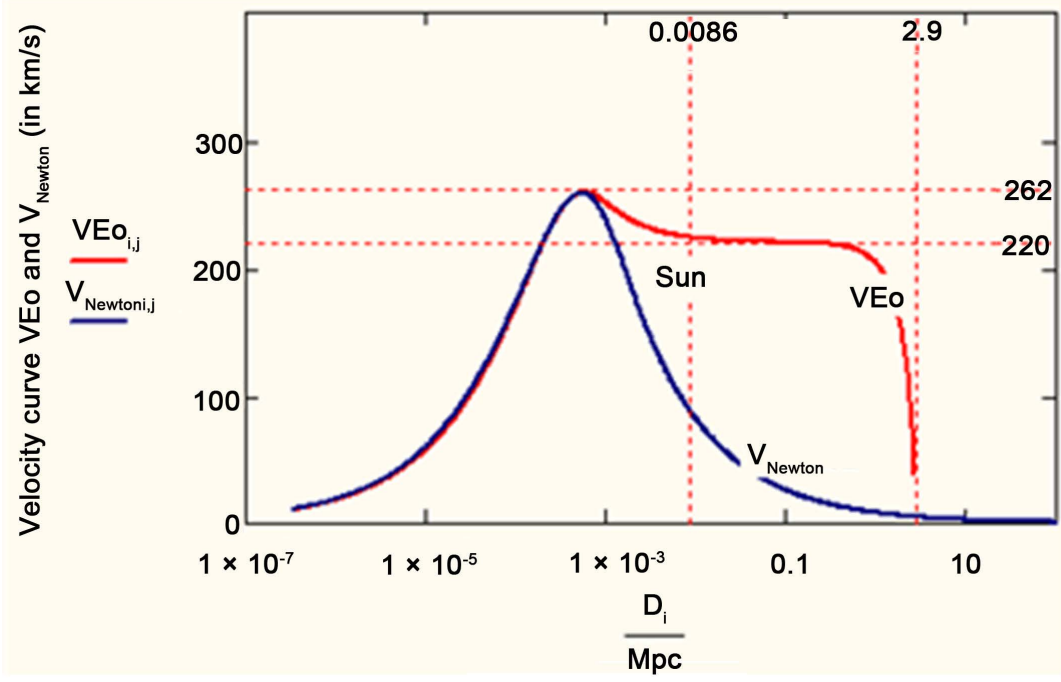

Figure 2. Flat velocity curve of the Milky way galaxy.
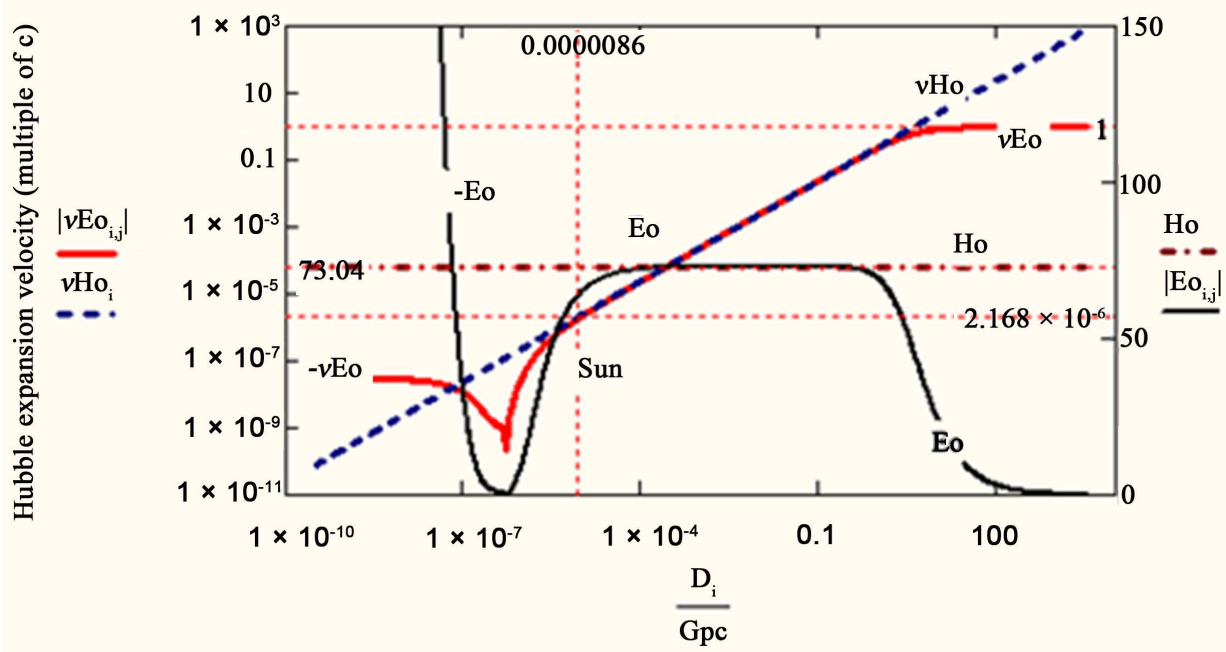

Distance D in Gpc

Figure 3. Relationship between the Hubble constant and $E_{0}$.

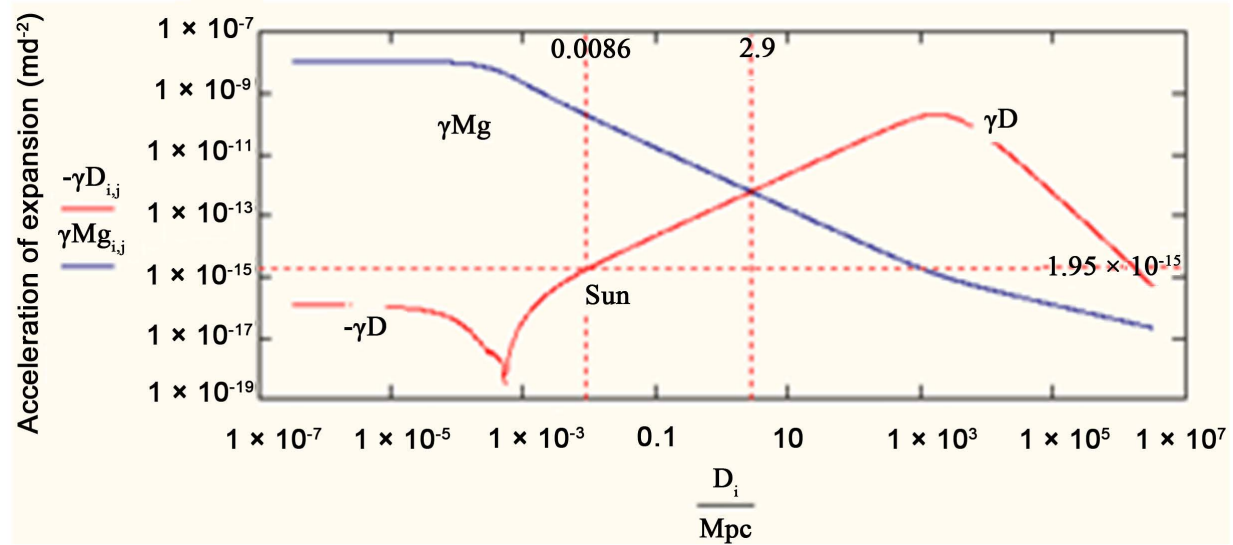

Figure 4. Acceleration of a celestial body of mass $M_{g}$ and acceleration of expansion of the universe $\gamma_{D}$. 
[6], and $E_{0}$ for a large distance ( $10 \mathrm{Mpc} \leq D \leq 50 \mathrm{Mpc}$ ) is equal to $73.036 \pm 0.1$ $\mathrm{km} \cdot \mathrm{s}^{-1} / \mathrm{Mpc}$. I stated the position of the Sun in the Milky Way, which is approximately equal to $8.6 \mathrm{pc}(0.0000068 \mathrm{Gpc})$ from the galactic center, with a rotation speed of 230 $\mathrm{km} \cdot \mathrm{s}^{-1}$ and an expansion rate of $2.168 \cdot 10^{-6} \mathrm{c}=0.650 \mathrm{~km} \cdot \mathrm{s}^{-1}$.

In Figure 3, we can see the difference between $H_{0}$ and $E_{0}$. For $H_{0}$, the speed $v H_{0}$ represents a straight line with a fixed slope, whereas for $E_{0}$, the slope is variable all along distance $D$. At large distances, the expansion rate $v E_{0}$ is positive (repulsion, i.e., galaxies move apart) and tends toward the speed of light $c$ (thus conforming to Einstein's relativity theory); at a smaller distance it becomes negative (attraction) and almost constant, for part of the curve after the reversal since the distance $0.5 \mathrm{kpc} \rightarrow 0 \mathrm{kpc}$. This part of the curve is at small distances less than $0.5 \mathrm{kpc}$, particularly regarding the solar system and its planets for which the expansion function $E_{0}$ also applies. I can also plot the curve of the acceleration of the expansion of the universe $\gamma_{D}$, taking the derivative of $E_{0} \cdot D$ with respect to time as

$$
E_{0} \cdot D \cdot \frac{\mathrm{d}\left(E_{0} \cdot D\right)}{\mathrm{d} D}=\gamma_{D}
$$

where $\mathrm{d} D=V \cdot \mathrm{d} t=E_{0} \cdot D \cdot \mathrm{d} t$ (Figure 4).

In Figure 4, we can see three areas of acceleration: a zone from 0 to $0.5 \mathrm{pc}$ where there is a constant rate of negative expansion (of $10^{-16} \mathrm{~m} \cdot \mathrm{s}^{-2}$, attraction); a zone between $0.5 \mathrm{pc}$ and $2 \mathrm{Gpc}$ where there is an accelerated rate of positive expansion (repulsion); and a zone between $2 \mathrm{Gpc}$ and above $3 \mathrm{Tpc}$ where there is a decelerated rate of expansion (always repulsive). Finally, the acceleration $\gamma_{D}$ produced by the expansion of the universe is much less than the acceleration $\gamma M_{g}$ produced by mass $M_{g}$ up to the value of $2.9 \mathrm{Mpc}$ for the Milky Way, and is negligible for our solar system. It seems that beyond $2.9 \mathrm{Mpc}$, other celestial bodies cannot be orbiting the Milky Way!

\section{Definition of the Value of the Speed of Reference $V_{0}$ in Our Solar System}

I can rewrite an approximation of Equation (8) by neglecting the acceleration of the universe for the Earth (Figure 4) and by setting $v=0$ as follows (here for our solar system):

$$
V_{\text {measured }}=V E_{0}=V_{\text {earth }}=\sqrt{\frac{G \cdot M g}{D} \cdot\left(\frac{1}{\sqrt{1-\frac{E_{0} \cdot D}{c}}}\right) \cdot \Lambda\left[W p e \cdot \frac{D}{r h}+1\right]}
$$

This reference speed $V_{0}$ will be calculated at the level of our solar system. This has two principal advantages: we know the precise Earth-Sun distance, and the flat expansion velocities are relatively stable (here attractive to the Sun; see Figure 3). Using universal Equations (8), (9), and (10), which also apply to our solar system, I deduce the value of $V_{\text {ref }}=V_{0}$, depending on the position and speed of rotation of the Earth around the Sun ( $D=R_{\text {earth-sun }}$ and $V_{\text {earth }}=V E_{0}$, respectively). By comparing the value of $E_{0}$, which is given by Equation (10), with the value in Equation (9), I get: 


$$
V_{0}=c \frac{\frac{V_{\text {earth }}^{2}}{G \cdot M g}-\frac{\Lambda}{D}\left(W p r \cdot \frac{D}{r H}+1\right)}{\Lambda \cdot\left(W p r \cdot \frac{D}{r H}+1\right)} \cdot Y \cdot D
$$

In this relationship the value of $Y$ is obtained by replacing the value of $V_{0}$ in Equation (9) by $Y \cdot E_{0} \cdot D$. Indeed, the $V_{0}$ value is similar at the speed of expansion of the universe, and this fact answers the classical formula of Hubble. The function $Y$ is a constant dependent on the zone of space considered. For a distance $D$ (about the distances of the solar system) $Y \approx 10$. Finally, the calculation of $V_{0}$ in the solar system gives $V_{0}=283.644 \mathrm{~m} \cdot \mathrm{s}^{-1}$. To obtain this value, $\mathrm{I}$ have to check the equation

$$
V_{0}\left(V_{\text {ref }}\right) / V_{\text {ref }}=1
$$

where $V_{0}=Y \cdot E_{0} \cdot D$ (Figure 5).

The term $\left(n_{0} / n\right)^{5}$ depends on the nature of the star, galaxy, or planet in question; here for our solar system $n=2.455388002$ and $n_{0}=2$, and for the Milky Way $n=2$ and $n_{0}=2$. The values of $n$ and no define the function $\Lambda$. Thus, the reference speed $V_{0}$ will have a value dependent on the nature of the considered system, or:

$$
V_{0}=V_{\text {ref }} \cdot\left(\frac{n}{n_{0}}\right)^{5} \text { with } V_{\text {ref }}=101.7 \mathrm{~m} \cdot \mathrm{s}^{-1}
$$

For the solar system: $V_{0}($ Sun $)=V_{\text {ref }} \cdot\left(\frac{n(=2.455388002)}{n_{0}(=2)}\right)^{5}=283.644 \mathrm{~m} \cdot \mathrm{s}^{-1}$.

In the Milky Way galaxy: $V_{0}=V_{\text {ref }} \cdot\left(\frac{n(=2)}{n_{0}(=2)}\right)^{5}=101.7 \mathrm{~m} \cdot \mathrm{s}^{-1}$.

Thus, $V_{\text {ref }}$ is a universal speed of reference for all gravitational systems. This value $V_{\text {ref }}$ is thus directly related to the value of the expansion rate of the universe $E_{0}$, as well as to the flat rotation curves of galaxies. Figure 6 shows the point where the distance of the Sun from the center of the Milky Way and the ratio $V_{0} / V_{\text {ref }}=\left(n / n_{0}\right)^{5}$

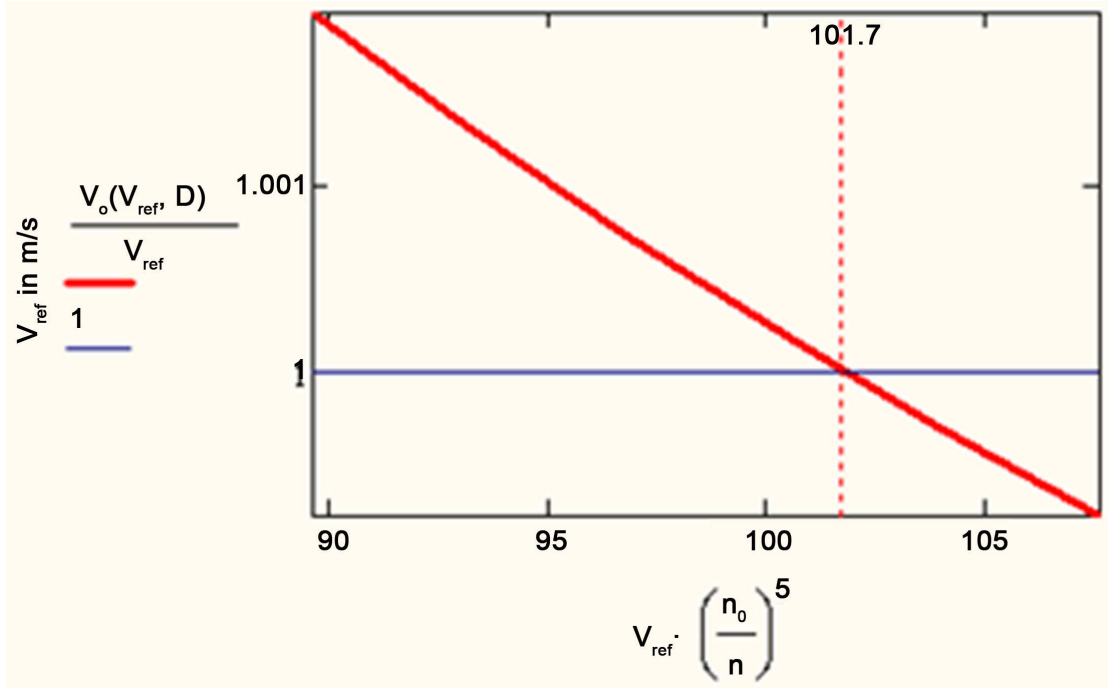

Figure 5. Ratio $V_{0} / V_{\text {ref }}$. 


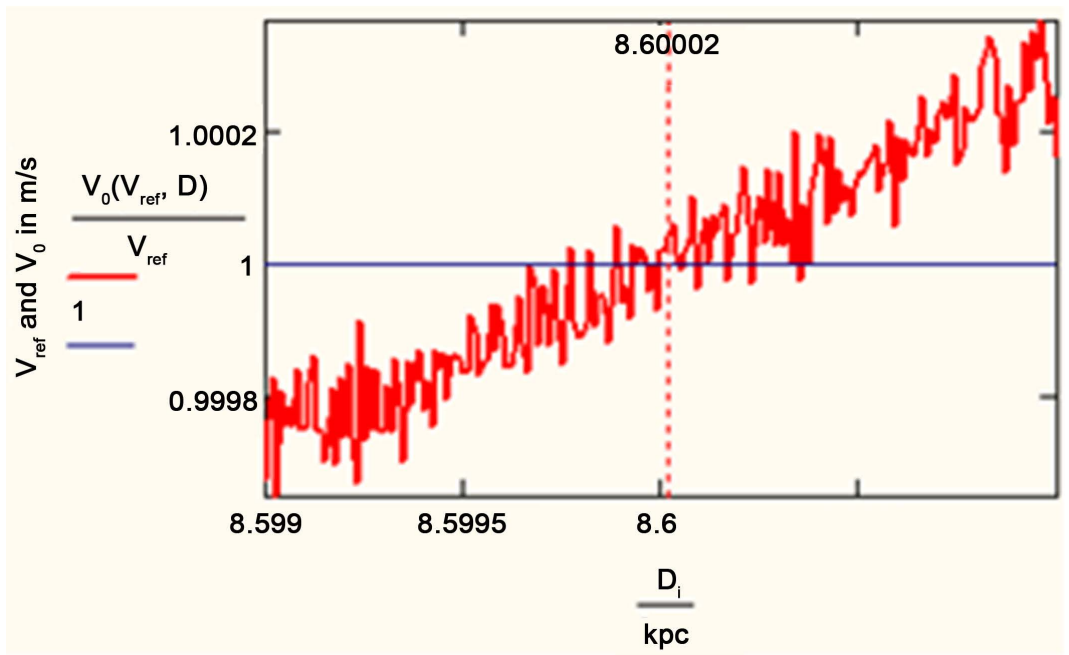

Figure 6. Distance from Sun to center of Milky Way for $V_{0} / V_{\text {ref }}=1$.

coincide. This equality requires the conditions $\frac{V_{0}\left(V_{\text {ref }}\right)}{V_{\text {ref }}}=1$ for $n_{0}=n$ and $D_{\text {sun }}$ to the center of the Milky Way equal to $8.60002 \mathrm{kpc}$.

\section{Conclusion}

I have shown in this article that it is possible to link the concept of expansion of the universe through a new term $E_{0}$ (which is comparable to the Hubble constant $H_{0}$ ), and the flat rotation curves of galaxies, with one limit according to galactic mass. For the Milky Way, the limit (end of the non-Newtonian rotation curves) is around 2.9 Mpc. This is possible using both the widely accepted gravitational theories of Newton and Einstein. I suggest a solution (Equations (8) and (10)) for the expression of the flat rotation speed of galaxies (or other galactic objects) which is derived from the universal relation of expansion of the universe $E_{0}$ (Equation (9)). This solution gives excellent results. The first and second derivatives of the value of $E_{0}$ (Equation (6)) are a function of time, and show that the universe is in acceleration until about $3 \mathrm{Mpc}$, then in deceleration until approximately $3 \mathrm{Tpc}$. This is new information about the universe. Thus, we can legitimately ask whether dark matter is necessary to explain the flat rotation curves of galaxies? And because of this accelerating expansion, could it be that dark energy does not exist? In this article, I have shown that the basic concept of the expansion of the universe also applies to the solar system like with planets and their satellites. Based on this, the Hubble constant $H_{0}$ and of Elbeze $E_{0}$ could be measured with more accuracy. The results presented in this article may solve some current issues such as the energy density of galaxies, their masses, and may help to solve the mystery of the formation of the universe and the presence of filaments of material connecting the clusters and super clusters of galaxies of the current standard model, all without the need to involve hypothetical dark matter and dark energy.

\section{References}

[1] Milgrom, M. (1983) Astrophysical Journal, 270, 365-370. https://doi.org/10.1086/161130 
[2] Einstein, E. (1950) The Meaning of Relativity. Princeton University Press, Princeton.

[3] Elbeze, A.C. (2012) Earth, Moon, and Planets, 108, 151-163. https://doi.org/10.1007/s11038-012-9388-9

[4] Elbeze, A.C. (2013) SpringerPlus, 2, 513. https://doi.org/10.1186/2193-1801-2-513

[5] Wendy, L., et al. (1994) Nature, 371, 757-762. https://doi.org/10.1038/371757a0

[6] Riess, A.G., et al. (2016) A 2.4\% Determination of the Local Value of the Hubble Constant. arXiv:1604.01424.

[7] Elbeze (2005) New Atomic Physics (NAP). 


\section{Appendix 1: Calculation of $\Lambda$}

Let $n=n_{0}=2$, a relative coefficient taken as a reference for our galaxy, the Milky Way. Any other object in the universe will also have a number $n$ that varies according to the object's characteristics such as its mass distribution, its linear density $\sigma$, and the shape of its flat rotation curves. For the Sun $n=2.455388002$. In this article, the distribution of the masses is comparable to that of a thin disk (the spherical distribution does not change the final results) of apparent mass equal to $M D$ :

$$
M D=\int_{0}^{D} 2 \pi r \cdot \sigma_{0} \cdot e^{-\frac{r}{R_{0}}} \cdot \mathrm{d} r
$$

where $D$ is the distance from the center of the object of interest (e.g., galaxy, star) and $R_{0}$ is the radius of the central core, where for a galactic disk such as that of the Milky Way, $R_{0}$ is generally taken as equal to $0.15 \mathrm{kpc}$, for the sun and the earth their radius. The density $\sigma_{0}$ is equal to $\sigma_{0}=$ Mass of the body $/ \mathrm{kpc}^{2}$.

The value of $\Lambda$ is defined as $\Lambda=\frac{M D}{M^{\prime} D}$, where $M^{\prime} D$ is the hidden value of the galactic mass near to its actual value for the distance $D$ being considered. Let this mass be equal to

$$
M^{\prime} D=\int_{0}^{D} 2 \pi r \cdot \alpha \sigma_{0} \cdot \mathrm{e}^{\frac{r}{\beta \cdot R_{0}}} \cdot \mathrm{d} r .
$$

I define the coefficients $\alpha$ and $\beta$ as being equal to

$$
\alpha=\frac{a}{n} \text { here } a=2.3 \quad \text { and } \beta=2 \cdot\left(\frac{n_{0}}{n}\right)^{3}
$$

and finally obtain $\Lambda$ as a function of distance $D: \Lambda=\frac{M D}{M^{\prime} D}$.

\section{Appendix 2: Calculation of Wpe}

In this article, the value of Wpe for our purposes is

$$
\mathrm{Wpe}\left(\mathrm{rH}, \mathrm{rH} \mathrm{H}_{0}, \mathrm{Ep}\right)=5.42 \cdot 10^{-30} \text {. }
$$

Here, Wpe is a function of at least three parameters: $r H$ is the atomic radius of hydrogen; $r H_{0}=r H \cdot n^{2}$ is the average atomic radius in the galaxy (or other object of interest) in terms of the orbital radius of hydrogen, where $n$ is defined in Appendix 1 ; and Ep is the electron rest energy.

The relationship giving Wpe is written as follows:

$$
\begin{gathered}
\text { Wpe }=\frac{1}{z e}-\frac{1}{z p} \\
z e=\sqrt{1+\frac{E \Delta p \cdot x p+E \Delta e \cdot x e}{E e}} \\
z p=\sqrt{1+\frac{E \Delta p \cdot x p+E \Delta e \cdot x e}{E p}} \\
x e=\left[\frac{2 \lambda e}{\Gamma \Delta e} \cdot \frac{r H}{r H_{0}}\right]^{3} \quad x p=\left[\frac{2 \lambda p}{\Gamma \Delta p} \cdot \frac{r H}{r H_{0}}\right]^{3}
\end{gathered}
$$


The value $(E \Delta p \cdot x p+E \Delta e \cdot x e)$ is always very small compared with $E p$ and $E e$ (proton and electron rest energy), and we can write:

$$
W p e=\frac{1}{z e}-\frac{1}{z p} \approx \frac{(E \Delta p \cdot x p+E \Delta e \cdot x e)^{2} \cdot\left(E e^{2}-E p^{2}\right)}{2 E e^{2} E p^{2}}
$$

where $E e$ and $E p$ are the electron and proton rest energies, respectively; $\lambda e$ and $\lambda p$ are modified wavelengths of the electron and the proton; $\Gamma \Delta e, E \Delta e$ and $\Gamma \Delta p$, $E \Delta p$ are the radial and volumetric interaction energy between the electron and the proton in the hydrogen atom. These values define the volume and the binding energy of the electromagnetic reaction between the electron and the proton, and ze and $z p$ are the energy charge (similar to electric charge) of the proton and electron in the hydrogen atom as follow table:

\begin{tabular}{cccc}
\hline Energy of electron and proton & $\lambda e$ and $\lambda p$ & $\Gamma \Delta e$ and $\Gamma \Delta p$ & $E \Delta e$ and $E \Delta p$ \\
\hline$E e=8.18711 \cdot 10^{-14} \mathrm{~J}$ & $2.82 \cdot 10^{-15} \mathrm{~m}$ & $7.3 \cdot 10^{-13} \mathrm{~m}$ & $3.76 \cdot 10^{-20} \mathrm{~J}$ \\
$E p=1.50328 \cdot 10^{-10} \mathrm{~J}$ & $1.53 \cdot 10^{-18} \mathrm{~m}$ & $1.7 \cdot 10^{-14} \mathrm{~m}$ & $8.86 \cdot 10^{-22} \mathrm{~J}$ \\
\hline
\end{tabular}

By using the values in table, we obtain a value for our galaxy the Milky Way of $W p e=5.42 \cdot 10^{-30}$. For a more detailed explanation of these values, the reader may wish to refer to the book by Elbeze [7].

In fact, for a first reading of this appendix, we can neglect the values of the above table and consider only the value of Wpe $=5.42 \cdot 10^{-30}$ as being a constant of integration.

Submit or recommend next manuscript to SCIRP and we will provide best service for you:

Accepting pre-submission inquiries through Email, Facebook, LinkedIn, Twitter, etc.

A wide selection of journals (inclusive of 9 subjects, more than 200 journals)

Providing 24-hour high-quality service

User-friendly online submission system

Fair and swift peer-review system

Efficient typesetting and proofreading procedure

Display of the result of downloads and visits, as well as the number of cited articles

Maximum dissemination of your research work

Submit your manuscript at: http://papersubmission.scirp.org/

Or contact jmp@scirp.org 